\title{
The Approach for Studying Variability of Sea Wave Spectra in a Wide Range of Wavelengths from High-Resolution Satellite Optical Imagery
}

\author{
Valery Bondur ${ }^{1}$ and Alexander Murynin ${ }^{1,2, *(\text { CD }}$ \\ 1 AEROCOSMOS Research Institute for Aerospace Monitoring, 105064 Moscow, Russia; \\ vgbondur@aerocosmos.info \\ 2 Federal Research Center "Computer Science and Control", Russian Academy of Sciences, \\ 119333 Moscow, Russia \\ * Correspondence: amurynin@bk.ru
}

Citation: Bondur, V.; Murynin, A. The Approach for Studying Variability of Sea Wave Spectra in a Wide Range of Wavelengths from High-Resolution Satellite Optical Imagery. J. Mar. Sci. Eng. 2021, 9, 823. https://doi.org/ 10.3390/jmse9080823

Academic Editor: Grigory Ivanovich Dolgikh

Received: 1 July 2021

Accepted: 26 July 2021

Published: 30 July 2021

Publisher's Note: MDPI stays neutral with regard to jurisdictional claims in published maps and institutional affiliations.

Copyright: (c) 2021 by the authors. Licensee MDPI, Basel, Switzerland. This article is an open access article distributed under the terms and conditions of the Creative Commons Attribution (CC BY) license (https:/ / creativecommons.org/licenses/by/ $4.0 /)$.

\begin{abstract}
The development and validation of a method for remote measurement of the spectra of sea waves, which significantly expands the capabilities to study surface waves in large water areas in a wide range of wavelengths, is described. The applied approach is based on the use of retrieving operators, which are constructed by the method of numerical simulation, taking into account the nonlinear nature of brightness field modulation by the slopes of the sea surface. Retrieving operators have a set of parameters that are adapted to the real conditions of aerospace imaging. To assess the adequacy of the retrieving of wave spectra recorded from satellite images, they are compared with the spectra obtained by ground-based means under controlled conditions. The studies have shown the adequacy of remote measurement of the spectra of slopes and elevations of sea waves with wavelengths in the range $0.1-1 \mathrm{~m}$. The possibility of using the developed method for studying the variability of sea waves in the coastal zone under conditions of limited fetch, including in the presence of anthropogenic disturbances, is shown.
\end{abstract}

Keywords: sea surface; wave spectra; satellite imagery processing; aerospace monitoring; sea waves; wave spectra; retrieving operator

\section{Introduction}

The spatiotemporal structure of surface waves changes randomly under the influence of many natural factors (internal waves [1], current fields [2,3], and anthropogenic impacts $[4,5])$ on the water environment. For a compact presentation of information about the structure of surface waves and its changes under the influence of various processes, it is advisable to use spatial and frequency spectra of sea waves, characterizing the distribution of wave energy over wave components of different lengths and periods [6,7]. An effective way to obtain information on the spatial spectra of sea waves in vast areas of the seas and oceans is the spatial spectral analysis of various aerospace images $[4,7,8]$.

Satellite optical images of high spatial resolution significantly expand the capabilities to study the spectra of surface waves in large water areas in a wide range of spatial frequencies.

For a reliable assessment of the spectra of surface waves from satellite optical images, special methods should be used to reconstruct the characteristics of sea waves from the characteristics of the brightness fields recorded by space sensors [9-14]. In this case, retrieving operators are used, which can be built on the basis of numerical modeling of various imaging conditions and characteristics of remote sensing equipment, including taking into account the nonlinear modulation of brightness fields by surface slopes $[9,13,14]$.

To assess the adequacy of the retrieval of wave spectra recorded from space images, it is necessary to compare them with the spectra obtained by standard wave recorders under controlled conditions. 
This paper describes a method for studying the spectra of sea waves in a wide range of wavelengths, retrieved from the spectra of optical satellite images of high spatial resolution (tens of $\mathrm{cm}$ ) using specially developed retrieving operators. They are compared with the spectra obtained by the array of string wave recorders by processing stereo images taken from an offshore platform, as well as with the results of measurements made using drifting wave buoys.

\section{Research Methods}

Let us consider an approach to studying the spectra of elevations and slopes of the sea surface.

Rough sea surface is a random field of elevations (wave applicates):

$$
z=\zeta(x, y, t)
$$

where $(x, y, z)$ is the rectangular Cartesian coordinate system, in which the plane $(x, y)$ coincides with the level of a calm (no waves) water surface, and $t$ is the time.

Fixing (1) at $t=t_{0}$, we obtain a two-dimensional random function:

$$
z=\xi(x, y)=\left.\zeta(x, y, t)\right|_{t=t_{0}}
$$

when studying surface waves using optical images, two-dimensional fields of signal are recorded corresponding to a fixed moment in time. Therefore, in this work, we will consider the representation of waves in the form (2).

Optical images of the sea surface are formed as a result of reflection and refraction of light according to the laws of geometric optics, as will be discussed below. Therefore, when analyzing the structure of the sea surface, along with the field of elevations $\xi(x, y)$, it is convenient to characterize the fields of slopes (or gradients) along the axes [9]:

$$
\xi_{\alpha}(x, y)=\frac{\partial \xi(x, y)}{\partial \alpha}, \alpha=x, y
$$

The field of slopes (gradients) of the sea surface in an arbitrary direction $\varphi$, taking into account (3), can be expressed by the following equation:

$$
\beta_{\varphi}(x, y)=\cos \varphi \xi_{x}(x, y)+\sin \varphi \xi_{y}(x, y)
$$

Taking into account the properties of the Fourier transform, it is possible to relate the power spectrum $\Phi_{\varphi}(\mathbf{k})$ of the slope field (4) with the elevation field spectrum $\Psi(\mathbf{k})$

$$
\Phi_{\varphi}(\mathbf{k})=\left(\cos \varphi k_{x}+\sin \varphi k_{y}\right)^{2} \Psi(\mathbf{k})
$$

This relationship (5) can be used to retrieve the elevation spectrum $\Psi(\mathbf{k})$ if the slope spectrum is measured experimentally, for example, from data extracted from an aerospace image:

$$
\Psi(\mathbf{k})=\frac{\Phi_{\varphi}(\mathbf{k})}{\left(\cos \varphi k_{x}+\sin \varphi k_{y}\right)^{2}}
$$

However, Equation (6) restores the spectrum of elevations not for all values $\mathbf{k}$, since for:

$$
k_{x} / k_{y}=-\operatorname{tg} \varphi
$$

the denominator goes to zero. Therefore, in the vicinity of the line (7) in the spectrum of slopes, there is a lack of information on the spectrum of elevations of the sea surface. For complete retrieval of two-dimensional wave spectra $\Psi(\mathbf{k})$ from optical images, a multi-position method is used $[13,14]$.

The method is based on the use of $M(M \geq 2)$ fragments separated within a large image, or using several images that differ in shooting conditions. From $\mathrm{M}$ fragments, slope spectra corresponding to different directions of the gradient can be retrieved.

With the spatial stationarity (ergodicity) of waves, the selected fragments correspond to the same spectrum of sea surface elevations $\Psi(\mathbf{k})$, which makes it possible to create a system of $\mathrm{M}$ equations: 


$$
\Phi_{m}(\mathbf{k})=\left(\cos \varphi_{m} k_{x}+\sin \varphi_{m} k_{y}\right)^{2} \Psi(\mathbf{k}) m=1, \ldots, M
$$

From this system, one can determine the desired two-dimensional wave spectrum $[13,14]$ :

$$
\Psi(\mathbf{k})=\frac{\sum_{m=1}^{M} \Phi_{m}(\mathbf{k})}{\sum_{m=1}^{M}\left(\cos \varphi_{m} k_{x}+\sin \varphi_{m} k_{y}\right)^{2}}
$$

Thus, to retrieve a two-dimensional spectrum of sea surface elevations, it is necessary to have slope spectra for two or more directions.

The required set of slope spectra can be obtained in various ways:

- Stereo imaging, when images from two (or more) cameras with different viewing directions of the surface are simultaneously recorded;

- Processing of several fragments of images obtained during aerial photography with a wide shooting angle, on the assumption of spatial stationarity (some kind of ergodicity) of waves within the surface area captured by the camera's field of view;

- Processing of multi-temporal surveys of the same area of the surface from different angles using the assumption of the stationarity of waves.

When processing a one-shot satellite image, none of the listed sets of conditions are realized. Therefore, the retrieval of the two-dimensional spectrum of sea surface elevations is possible only outside the angular zone in the vicinity of the straight line defined by the condition (7).

In order to obtain the spectra of the slopes of the sea surface, retrieving operators $R(\mathbf{k})$ should be used, which make it possible to switch from the spectrum $S(\mathbf{k})$ of a fragment of the optical image to the spectrum of slopes $\Phi(\mathbf{k})$ :

$$
\Phi(\mathbf{k})=R(\mathbf{k}) S(\mathbf{k})
$$

In the early works devoted to this problem, a simple representation of the retrieving operator was based on the concept of a linear relationship between the surface slopes and the brightness recorded in the pixels of the images [15-17]. The concept of linear modulation of the brightness field by the slopes of the sea surface makes it possible to obtain a retrieving operator that does not depend on the wave vector: $R(\mathbf{k})=$ const.

In reality, the modulation of brightness by slopes can be significantly nonlinear, especially in the presence of sun glare.

From a physical point of view, the most important point is the distortion of the power exponent (slope) of the spectrum. Numerical estimates of such distortions were obtained in $[14,18]$. The adequacy of the proposed method for retrieving wave spectra from the spectra of optical images, which takes into account the nonlinear modulation of the recorded brightness field by the slopes of the rough sea surface [13,14], was first experimentally confirmed by experiments on an oceanographic platform near the village of Katsiveli (Black Sea) [19] and confirmed in subsequent works as applied to satellite images of high spatial resolution [9-11]. The adaptation of the method to the conditions of analysis of vast ocean areas from space images was carried out using the results of sea truth buoy measurements [20].

When constructing operators that retrieve the spectra of the sea surface slopes, it is necessary to take into account all the known physical mechanisms of non-linear distortions of the sea surface slope spectra $[13,18,21-24]$. Since it is difficult to use the relation between slope fields, sea surface brightness fields and their spectra for analytic solution of the problem, it is reasonable to conduct direct numerical modeling of the sea surface images and the calculation of the resulting distortions of the wave spectra.

A diagram illustrating the interaction of various algorithms for numerical modeling and image processing is shown in Figure 1.

To obtain the retrieving operators by the numerical method, the following sequence of operations is performed. 


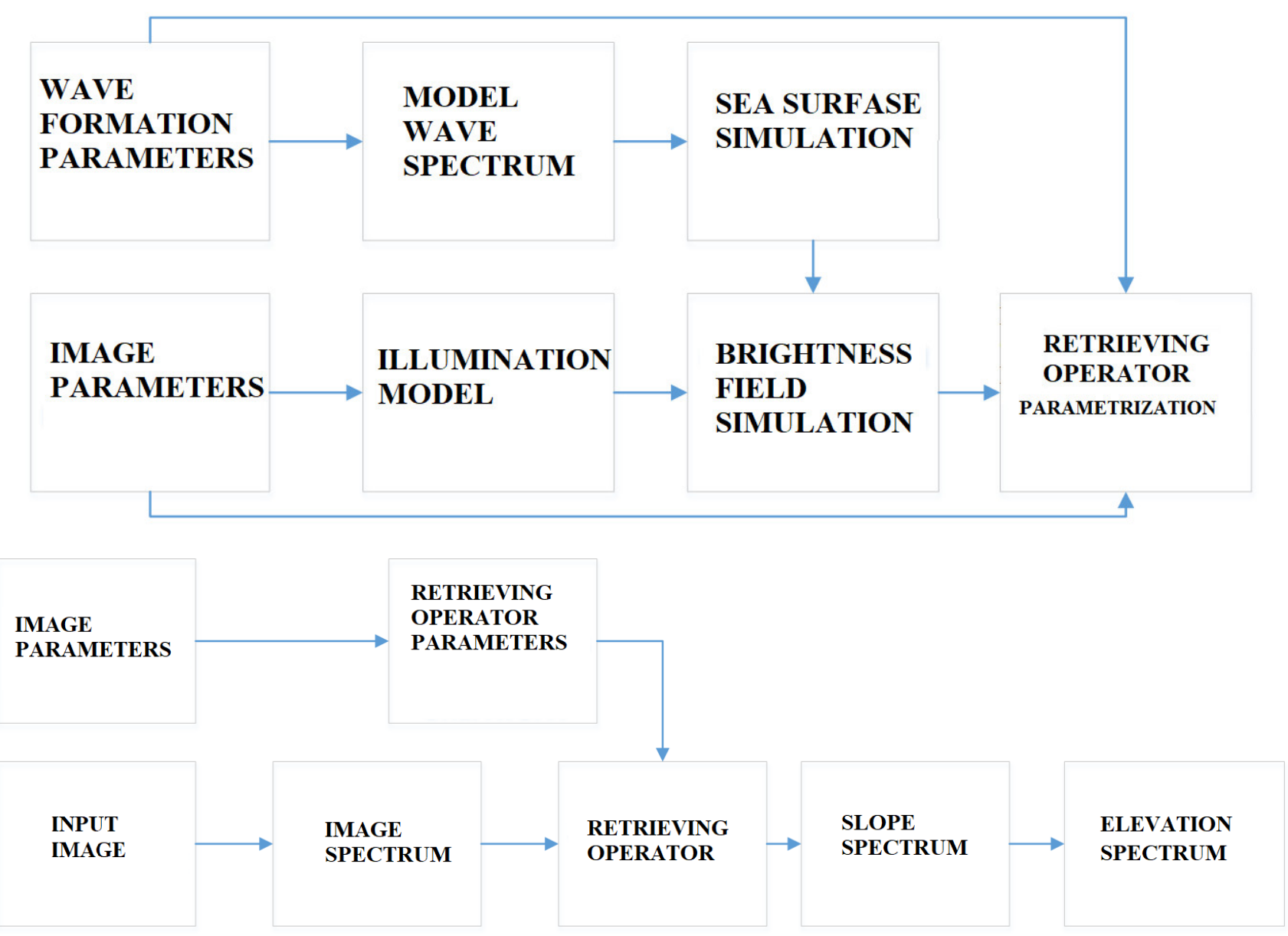

Figure 1. Diagram of interacting algorithms used to construct the retrieving operator by numerical simulation and processing satellite images using this operator.

Two-dimensional random fields of sea surface slopes $\xi_{\alpha}(x, y), \alpha=x, y$ are synthesized for a given spatial spectrum of elevations $G(\mathbf{k})$ and a phase spectrum given by a random function with a uniform distribution of phases $[9,13,14]$.

For the given illumination and viewing conditions, the brightness field, $L(x, y)$ is calculated using the synthesized slope field and taking into account various physical processes involved in its formation. When modeling optical images, a model is used that describes the reflection and refraction of light on the water surface according to the laws of geometric optics, as shown in Figure 2a. Figure 2a shows vectors of unit length that determine the reflection and refraction of light on an element of the sea surface according to the laws of geometric optics:

- the vector $v$ defines the normal to the sea surface element and depends on the gradient of the elevation field;

- vectors $\mathbf{r}_{1}$ and $\mathbf{r}_{2}$, indicate the directions in the upper and lower hemispheres, whence comes the light that enters the receiver after reflection and refraction, respectively;

- the vector $\mathbf{r}_{\odot}$ determines the direction to the Sun;

- vector $\mathbf{r}_{S}$ defines the direction to the sensor.

To take into account the nonlinearity of the modulation of the brightness field by the surface slopes due to the presence of sun glare, light scattering by small-scale surface roughness within the area, the size of which is determined by the spatial resolution of the sensor registering the image, is of significant importance. The higher the spatial resolution a satellite image has, the smaller the site size is. Accordingly, the dispersion of roughness is less and the effect of sun glare is greater. 


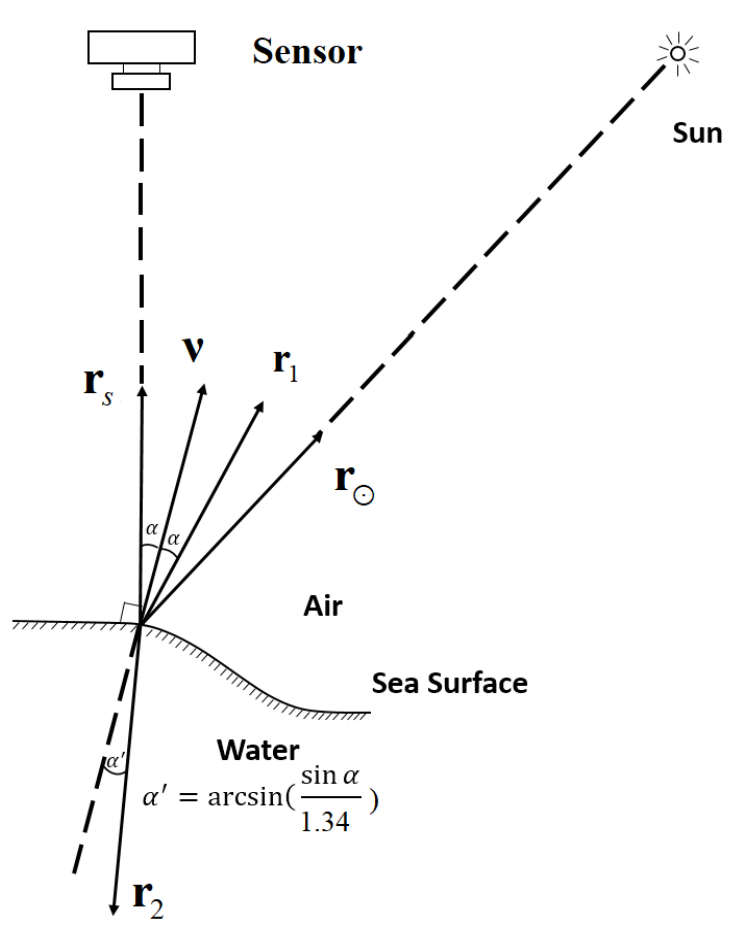

(a)

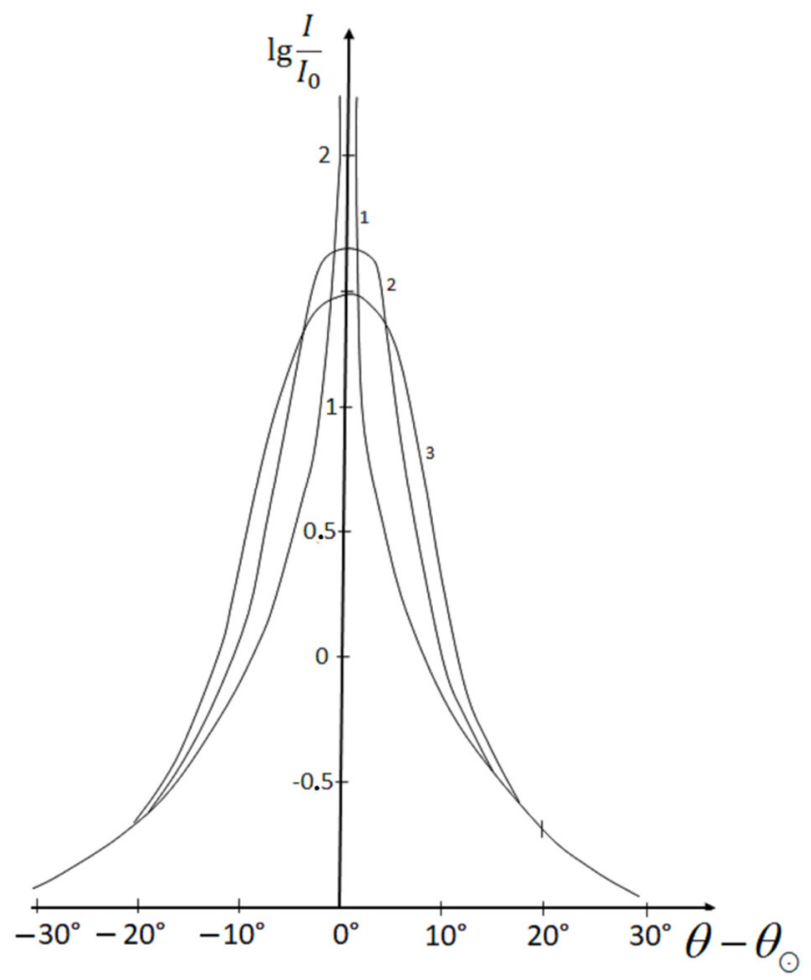

(b)

Figure 2. (a) Scheme of reflection and refraction of light at the air-water interface. The vectors of unit length indicate the directions in the upper and lower hemispheres from which light comes, falling into the receiver after reflection and refraction; (b) Angular distribution of the effective intensity of light in the vertical plane of the Sun, reflected by an element of the sea surface, for different sizes of this element (explained in the text).

Figure $2 b$ shows examples of the angular dependence of the effective light intensity in the plane of the solar vertical, corresponding to different image pixel sizes $\Delta$ and rms roughness $\sigma_{\Delta}: 1-\Delta \rightarrow 0, \sigma_{\Delta}=0 ; 2-\Delta=0.1 \mathrm{~m}, \sigma_{\Delta}=0.07 \mathrm{rad} ; 3-\Delta=1 \mathrm{~m}, \sigma_{\Delta}=0.09 \mathrm{rad}$ [18]. Light intensity is normalized using a multiplier used to approximate the sky light intensity distribution on a cloudless day $[18,25]$.

The brightness field, $L(x, y)$ is converted into a model image signal, $L_{m}(x, y)$, taking into account the specified characteristics of the recording equipment.

The retrieving operator $\mathrm{R}$ is defined by comparing the spectrum of the model optical image, $S_{M}(\mathbf{k})$ to the spectrum of slopes $\Phi_{G}(\mathbf{k})$ corresponding to the direction $\varphi_{c}$ determined by the specified illumination and viewing conditions. In particular, the retrieving operator can be represented in the form of a spatial-frequency filter $[9,13,14]$.

$$
R(\mathbf{k})=\frac{\left(\cos \varphi_{c} k_{x}+\sin \varphi_{c} k_{y}\right)^{2} G(\mathbf{k})}{S_{M}(\mathbf{k})}
$$

With this definition of the retrieving operator, the retrieval of the sea surface slope spectrum $\Phi(\mathbf{k})$ is reduced to multiplying the spectrum of the image fragment, $S(\mathbf{k})$ by the spatial frequency filter, $R(\mathbf{k})$ according to Equation (10).

When numerically simulating images of the sea surface, parameters must be set that determine the conditions for the formation of these images, and therefore, to one degree or another, affect the type of the retrieving operator $R(\mathbf{k})$. When processing real images of the sea surface, these parameters are taken directly from the conditions for obtaining an optical image.

When analyzing large amounts of information to determine the retrieving operator, $R(\mathbf{k})$, it is advisable to perform preliminary numerical modeling of sea surface images for various combinations of parameters. For each specific combinations of parameters retriev- 
ing operator $R(\mathbf{k})$ is approximated by an analytical formula $R(\mathbf{k})=R(\mathbf{k}, \mathbf{a})$ associated with a certain set of parameters $\mathbf{a}$.

To parameterize the retrieving operator constructed by the numerical simulation, the power function of the wave number with parameters depending on the wave azimuth can be used as follows [11]:

$$
R(\mathbf{k}, \mathbf{a})=R_{l o}(\mathbf{k})\left(\cos \left(\phi-\phi_{c}\right)^{a_{3}} k^{-\left(a_{1}+a_{2} \cos \left(\phi-\phi_{c}\right)\right)}\right)
$$

where the parameters $a_{1} a_{2} a_{3}$ characterize the region of high spatial frequencies, where a power-law decay of the spatial wave spectrum is observed. For typical conditions of highresolution satellite imagery at different positions of the Sun, the values of these parameters were obtained by numerical simulation of physical processes of formation of optical images of waves $[9,18]$;

$R_{l o}(\mathbf{k})$ is the retrieving operator in the low-frequency region, the construction technique of which is described in [11]. In the same work [11], the results were obtained on preliminary parameterization of $R_{l o}(\mathbf{k})$.

- Thus, the practical use of the retrieving operator is as follows. From the conditions for obtaining images, a multi-dimensional vector of conditions is constructed, the components of which serve to determine the set of parameters a from the results of numerical simulation carried out in advance. The spatial-frequency filter constructed from the set of parameters $\mathbf{a}$ is then applied to the spatial spectra of the selected image fragments to calculate the corresponding spectra of the sea surface slopes. The obtained slope spectra are used to retrieve the elevation spectra:

- by Equation (9) in the presence of several spectra of slopes corresponding to different directions;

- $\quad$ or by Equation (6), taking into account the restrictions imposed by Equation (7), if there is no possibility of obtaining slope spectra for several different directions.

\section{Results}

To validate the developed method, an experimental check of the adequacy of its work should be carried out. The main focus of attention should be the validation of the method with the variability of waves associated with the presence of natural and anthropogenic anomalies.

To validate the retrieving operators constructed by the method of numerical simulation, we will use an approach based on the comparison of satellite and in situ measurements obtained in complex experiments [10]. Complex experiments are carried out under controlled conditions and include a space survey of test areas of the sea surface and synchronous sea truth measurements using wave recorders, as well as stereophotogrammetric survey of the sea surface at low altitude. Spatial spectra of sea surface elevations, retrieved from satellite images under fixed conditions, are compared with the spectra of elevations obtained from in situ measurements with string wave recorders using a special technique that takes into account the different nature of remote and in situ measurements [10].

To solve the set tasks, the results of complex experiments were used, which were carried out near the stationary oceanographic platform of the Black Sea hydrophysical sea truth test site (Katsiveli village, Crimea), installed at a distance of about $600 \mathrm{~m}$ from the coast.

Complex experiments of two types were carried out:

The main complex experiment (experiment \#1) consisted of a space survey and synchronous in situ measurements of wave spectra from the oceanographic platform.

An additional complex experiment (experiment \#2) consisted of conducting the research in the short-wave region of wave spectra $(\Lambda=0.04-1.0 \mathrm{~m})$ using string wave recorders and stereo imaging from the deck of an oceanographic platform under wave formation conditions close to those observed in the main complex experiments.

To measure the spatio-temporal wave spectra, we used in situ data obtained using a wave measuring complex based on an array of string wave recorders, which are a set 
of six resistive wave recorders measuring sea surface elevations at points located in the center and vertices of a pentagon. The distance from the central string to each of the outer strings is $25 \mathrm{~cm}$. The main technical characteristics of this complex: the maximum height of the measured waves - up to $4 \mathrm{~m}$; resolution — not worse than $3 \mathrm{~mm}$; number of measuring channels—6; and channel sampling frequency-10, 20, 50, and $100 \mathrm{~Hz}$.

\subsection{The Results of Studies of the Variability of Waves in the Complex Experiment \#1}

The purpose of the main complex experiments is to validate the method of remote measurement of sea wave spectra from satellite images using in situ measurements on a stationary platform.

The image was recorded by high spatial resolution equipment from the GEOEYE satellite on 24 September 2015 at 11:52 local time.

The details of processing the satellite image obtained in the main complex experiment are described in [10,11].

Satellite images were processed using the following sequence of computational operations:

- $\quad$ calculation of the spatial spectrum of the selected image fragment $S(\mathbf{k})$;

- retrieval of the spectrum of slopes $\Phi(\mathbf{k})$ using the retrieving operator $R(\mathbf{k})$, built for a set of conditions determined by the conditions for obtaining a space image;

- calculation of the spatial spectrum of elevations $\chi(k)$ from the retrieved spectrum of slopes in a given range of wave azimuths ( \pm 60 angular degrees relative to the azimuth of the Sun) according to formula (6);

- recalculation into the frequency spectrum $\psi(\omega)$ taking into account the dispersion ratio of gravitational waves by the formula:

$$
\psi(\omega)=\chi(k(\omega)) \frac{d k(\omega)}{d \omega}
$$

An example of the comparison of spectra is shown in Figure 3. Figure 3a shows a fragment of a high-resolution satellite image, registered from the GEOEYE satellite. The oceanographic platform from which in-situ measurements were carried out is marked with the yellow circle. The red rectangle marks a sea surface area used for the wave spectra retrieval.

Figure $3 \mathrm{~b}$ shows a sequence of spectra based on the data of a string wave-recorder, which demonstrates the variability of waves in the experimental area for three hours before satellite imagery. Each given spectrum was obtained with a half-hour averaging. The time evolution of the frequency spectra for three hours preceding the satellite's pass shows the development of wind waves-an increase in their energy and a decrease in the frequency of the spectral peak. At the same time, the system of swell waves remained dominant. Swell waves came from an azimuth of $130^{\circ}$, wind waves propagated along the direction of the east wind.

Figure $3 \mathrm{c}$ shows the spectrum of slopes, retrieved by the method described above from high-resolution satellite imagery data, and also shows the angular sector in which the spectrum of sea surface elevations was reconstructed for subsequent comparison with in situ measurements.

Figure $3 \mathrm{~d}$ combines the frequency spectra of elevations obtained during processing of the satellite image, taking into account the dispersion relation and the calculation by Equation (13), as well as the spectra obtained from the data of the array of string wave recorders. The measure of the discrepancy between the estimates of the spectra was $\Delta \approx 0.07$ [10].

The red dashed lines show the limits within which the spectrum of developing wind waves should lie according to the Toba approximation of the wave frequency spectrum [26]:

$$
S(\omega)=\alpha g u_{*} \omega^{-4}
$$

where $u_{*}$ is the dynamic speed in air, and $\alpha$ is the coefficient for two lines, equal to 0.06 and 0.11 , respectively. 


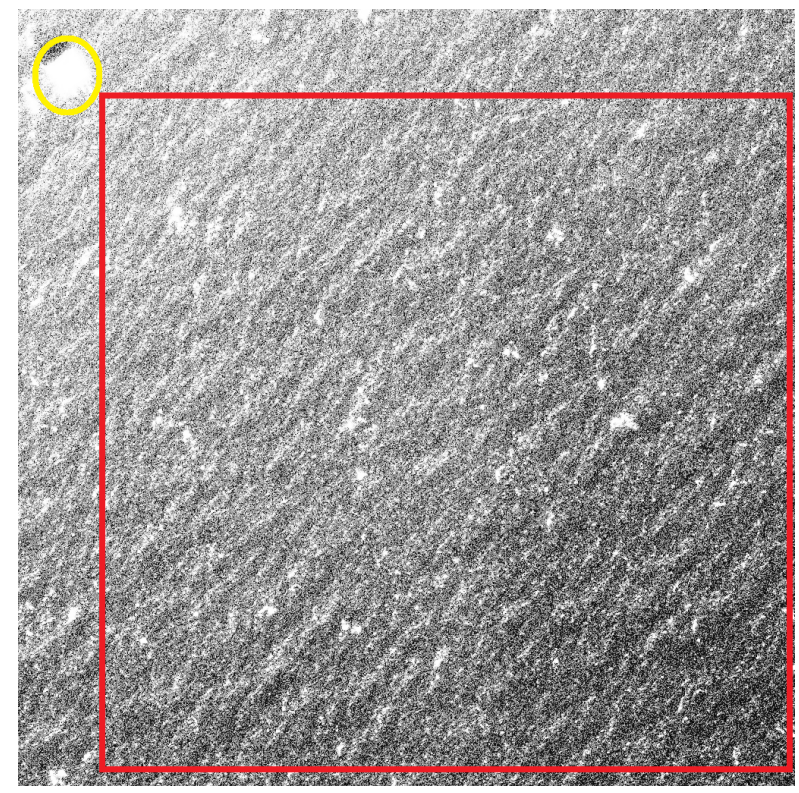

(a)

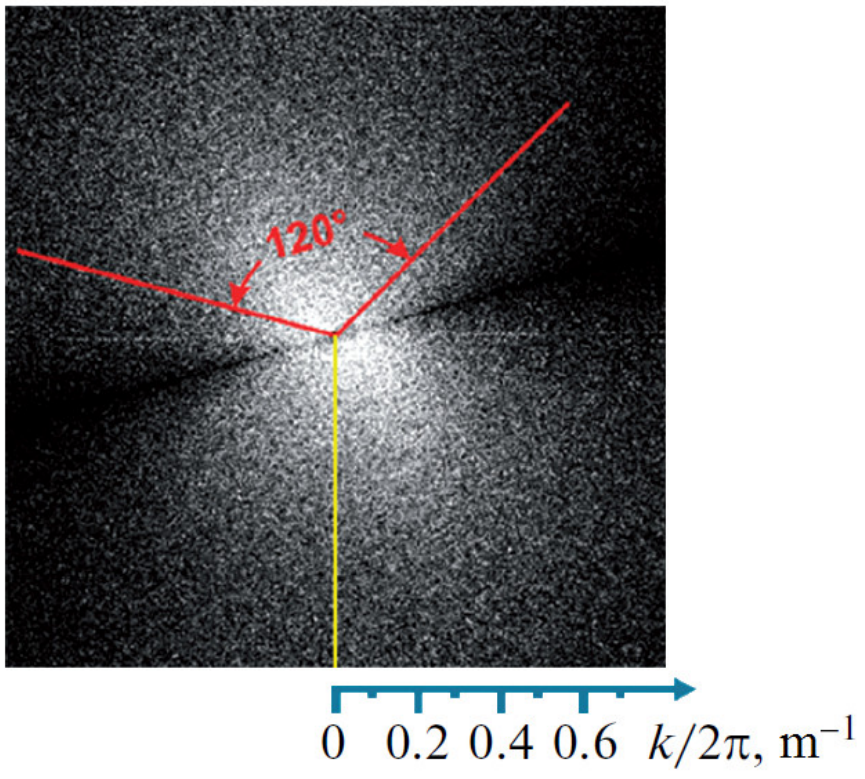

(c)

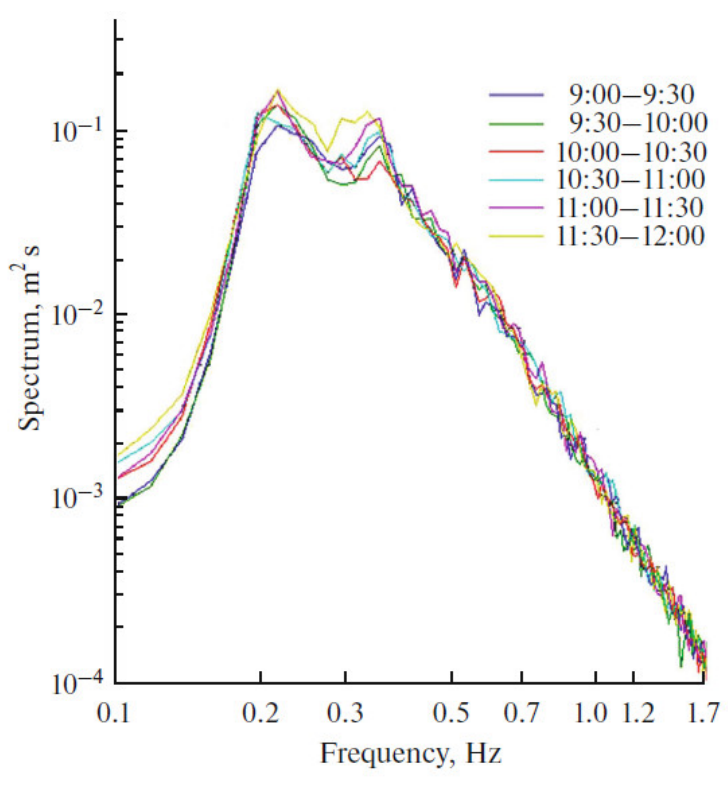

(b)

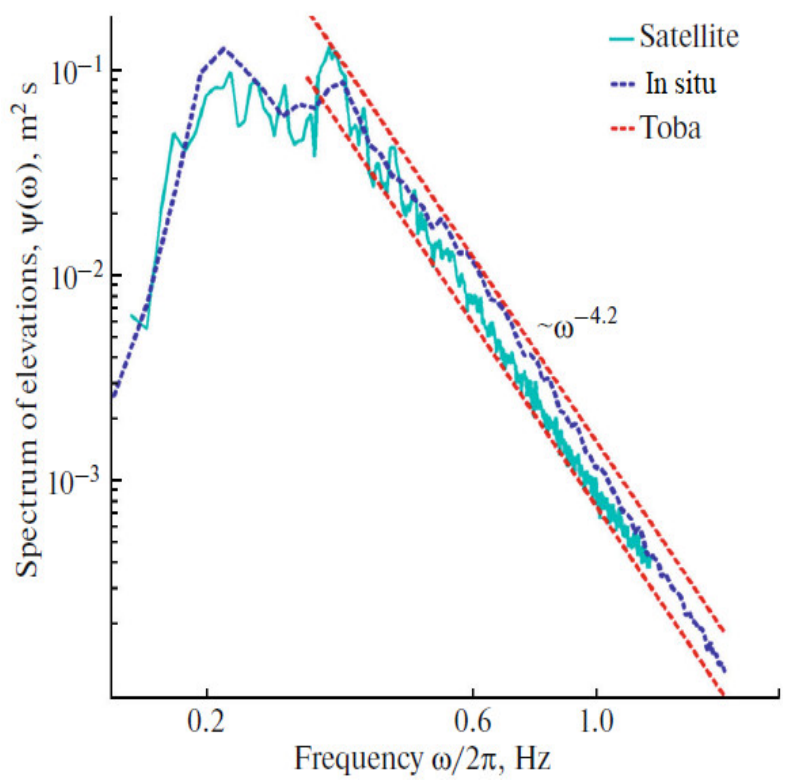

(d)

Figure 3. Comparison of sea wave spectra: (a) a tile of a satellite image, where a red rectangle marks a sea surface area with a linear size of $1 \mathrm{~km}$ used for the wave spectra retrieval, and the yellow circle marks the oceanographic platform from which in-situ measurements were carried out $(\mathbf{b})$ a sequence of spectra based on wave-recorder data for three hours with a half-hour averaging; (c) a slope spectrum with an outlined corner sector to retrieve a two-dimensional elevation spectrum; (d) comparison of spectra converted to frequency coordinates.

Figure 4 shows an example of comparing the frequency spectrum of elevations obtained by a half-hour averaging of the data of the array of string wave recorders with the spectra of elevations reconstructed by processing satellite image fragments of various sizes. The given plots show that when retrieving wave spectra from an image of a sea surface area with a linear size of $0.5 \mathrm{~km}$ (Figure $4 \mathrm{a}$ ), it is not possible to retrieve the spectrum of elevations in the low-frequency region due to the lack of data. 


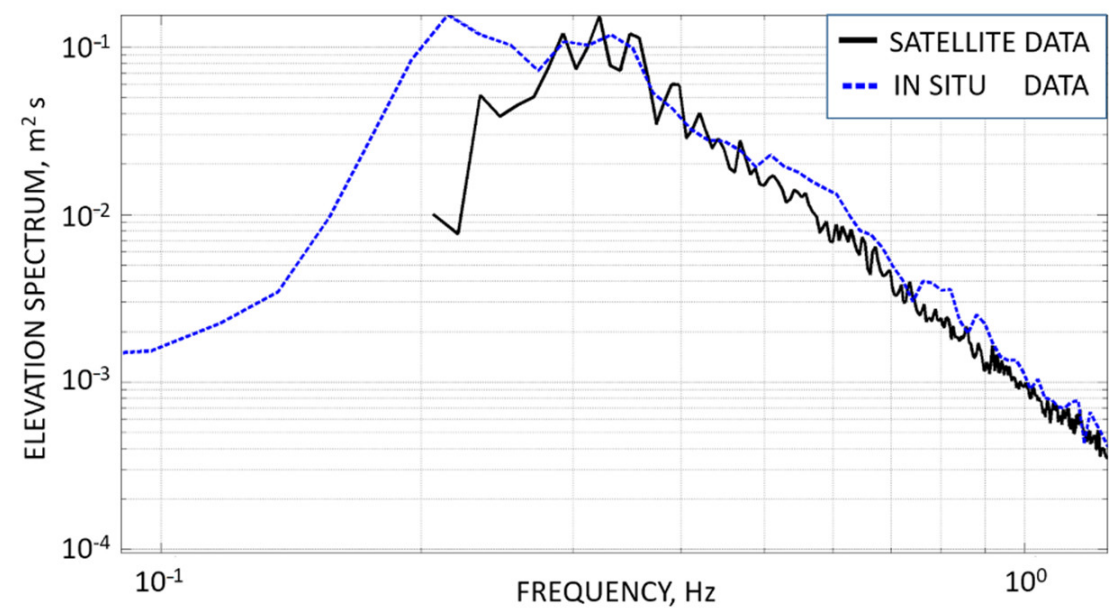

(a)

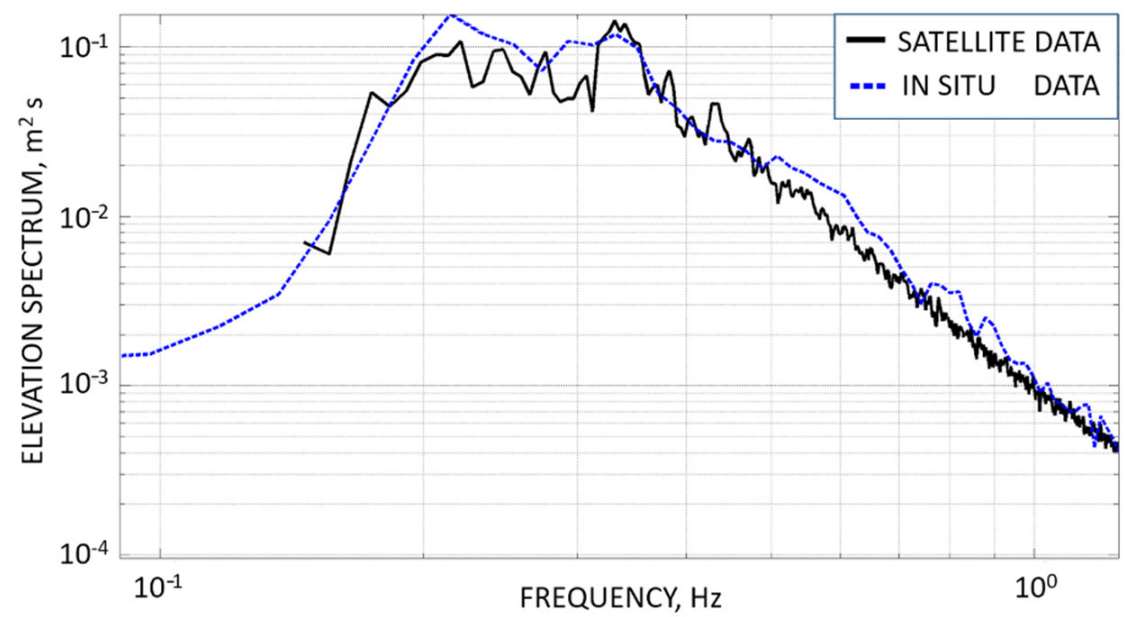

(b)

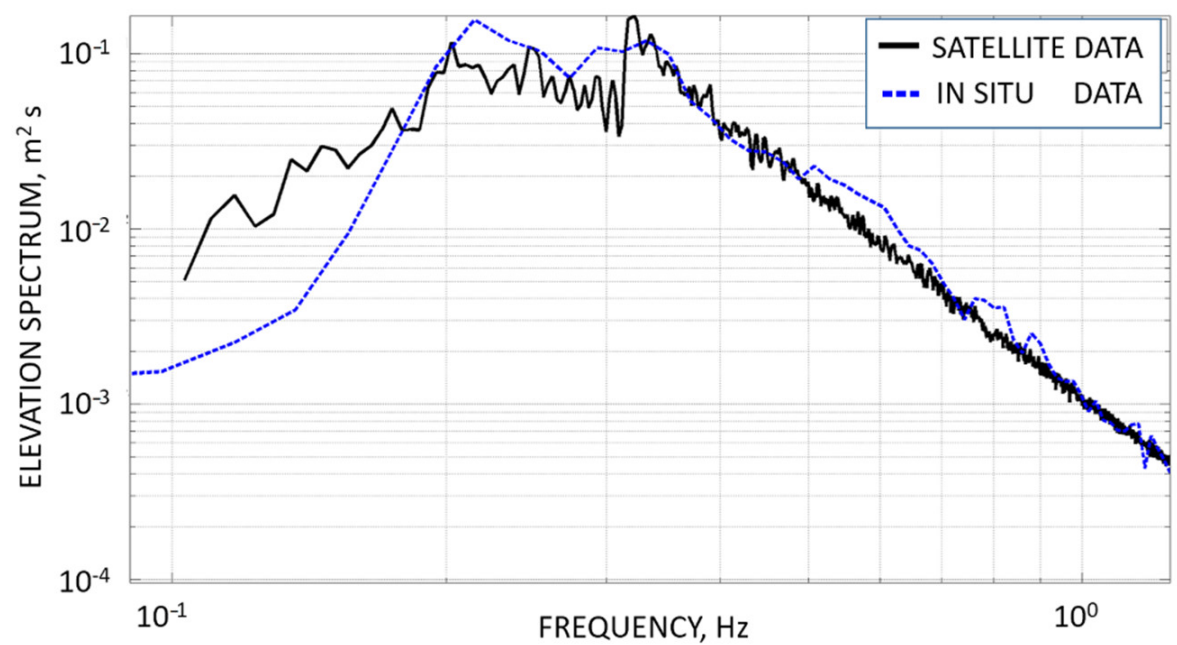

(c)

Figure 4. Comparison of the frequency spectra of sea wave elevations measured using the data of the string wave recorder with averaging over $30 \mathrm{~min}$ (immediately before satellite imagery) with the spectra retrieved from two-dimensional spectra reconstructed from satellite images at various linear dimensions of the sea surface area: (a) $0.5 \mathrm{~km},(\mathbf{b}) 1 \mathrm{~km}$ (c) $2 \mathrm{~km}$. 
When the spectrum of elevations is reconstructed from a region of the surface with a linear size of $2 \mathrm{~km}$, the retrieved spectrum turns out to be broader in the low frequency region than the spectrum obtained from the data of the array of string wave recorders. In this case, the processed area of the sea surface covers a zone remote from the coast, where the waves are more developed than in the zone where the string wave recorders are located. The best coincidence of the shape of the spectra is observed at a fragment linear size of $1 \mathrm{~km}$.

\subsection{The Results of Studies of the Variability of Waves in Complex Experiment \#2}

The purpose of the additional complex experiments is to study the applicability of the developed method for remote measurement of the spectra of sea waves in the region of short waves.

In additional experiments, along with in situ measurements carried out by string wave recorders, a stereo survey was carried out on the platform from a low altitude with a high spatial resolution.

Stereophotogrammetric processing of synchronously recorded optical images of the sea surface from two different points of view (stereopairs) makes it possible to directly measure the realizations of two-dimensional fields of sea surface heights, and from them to calculate the spatial spectra of elevations. The stereophotogrammetric method makes it possible to measure the shape of a rough sea surface and represent it as a function of Equation (2).

Stereo photography of the sea surface was carried out from the working platform, which was located at an altitude of $16 \mathrm{~m}$ above sea level with a baseline of $10.2 \mathrm{~m}$ using stereo cameras with a focal length of $99 \mathrm{~mm}[10]$.

Measurements of coordinates $x, y, z$ of sea surface elements by stereo-pairs. From the elevation field measurements, the spectra of the sea surface elevations were calculated $\Psi_{\varphi}\left(k_{\varphi}\right)$ using the interpolation procedures and the discrete Fourier transform.

Simultaneously with the stereophotography on the platform, measurements of the frequency spectra of the sea surface elevations were carried out with the help of a spectral wave recorder, which provided the registration of the spectrum in the frequency range, and the recording time of one spectrum in this range was about $20 \mathrm{~min}$.

Experimental conditions: the zenith angle of the Sun was $30^{\circ}$ (the images were recorded in a cloudless sky), and the wind speed was $5 \mathrm{~m} / \mathrm{s}$. During the experiments, the wind was blowing off the coast, so the fetch of the wind waves did not exceed the distance from the platform to the coast, which is $\sim 600 \mathrm{~m}$.

Images of the sea surface obtained during stereo photography were also used to retrieve the spectrum of the sea surface elevation using a developed retrieving operator. At the same time, fragments of two optical images of a stereopair were analyzed, in which the same area of the sea surface is presented, which was processed stereophotogrammetrically. This makes it possible, when comparing the estimates of the spectrum of sea surface elevations obtained by different techniques, to study the accuracy of these techniques.

Synchronous wave-recorder measurements were carried out to analyze the selected stereopairs. All the optical images under study were recorded under a cloudless sky.

Comparison of the estimates of the wave spectra obtained by different techniques was carried out in order to check the adequacy of the detection and measurement of variations in the wave spectra caused by the variability of the waves, associated both with natural processes and with anthropogenic influences.

The correlation between variations in the estimates of wave spectra obtained by different techniques was studied in [10]. We investigated the correlations of the main characteristics of the wave spectra obtained by three methods in two wavelength intervals: from 0.1 to $1 \mathrm{~m}$ and from $0.04 \mathrm{~m}$ to $0.4 \mathrm{~m}$.

For the main characteristics to compare the wave spectra obtained by different techniques, we chose the rms elevation and the exponent of the power-law approximation of the wave spectrum in the indicated intervals. 
The analysis of the obtained results showed that the correlation of estimates made by different techniques is quite high, and averages 0.8-0.9.

To check the adequacy of the developed techniques when working with the regions of the sea surface, where the wave spectrum is subject to anthropogenic impacts, an artificial anomaly of the spatial wave structure was created during the experiment using vegetable oil spilled onto the water.

The wave spectra obtained by different techniques in the area of the artificial anomaly were compared with similar spectra obtained earlier for the background sea surface.

The study of the background sea surface was carried out for two hours.

After that, an artificial anomaly was created by spilling about a liter of vegetable oil from the oceanographic platform. The creation of artificial films of surfactants has proven itself well as a method for studying anthropogenic pollution of the marine environment [22,27-29].

As a result of spilling the vegetable oil, an artificial slick was formed on the water. The area of this slick exceeded the water area under the platform. Special measurements of the thickness of the oil film on the water were not carried out. According to the estimates based on the volume of spilled vegetable oil and the approximate area of the slick, the film thickness was about $\sim 10^{-6} \mathrm{~m}$.

The artificial anomaly created with the spilled vegetable oil was investigated for about half an hour using three techniques.

Wave spectra measured by different methods were recalculated into the spectrum of elevations according to Equation (13).

According to our data, there is a noticeable damping of waves with lengths of 0.3-0.6 $\mathrm{m}$, as well as shorter waves. Waves longer than one meter are not considered. The spectra in Figure 5 have single measurements with wavelengths over $0.5 \mathrm{~m}$, for which the spectral power has decreased by about 2 times.

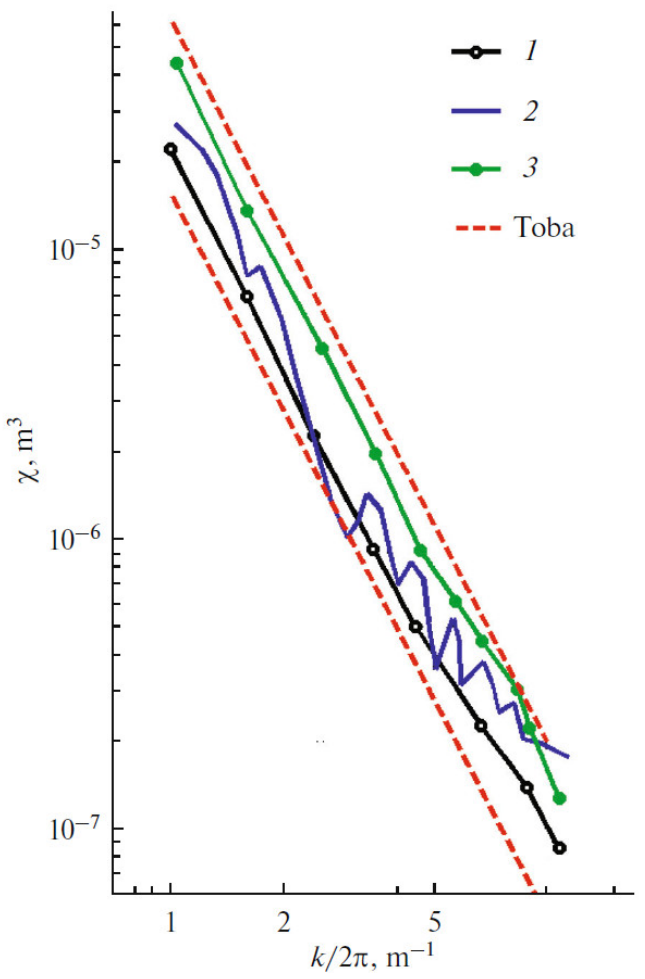

(a)

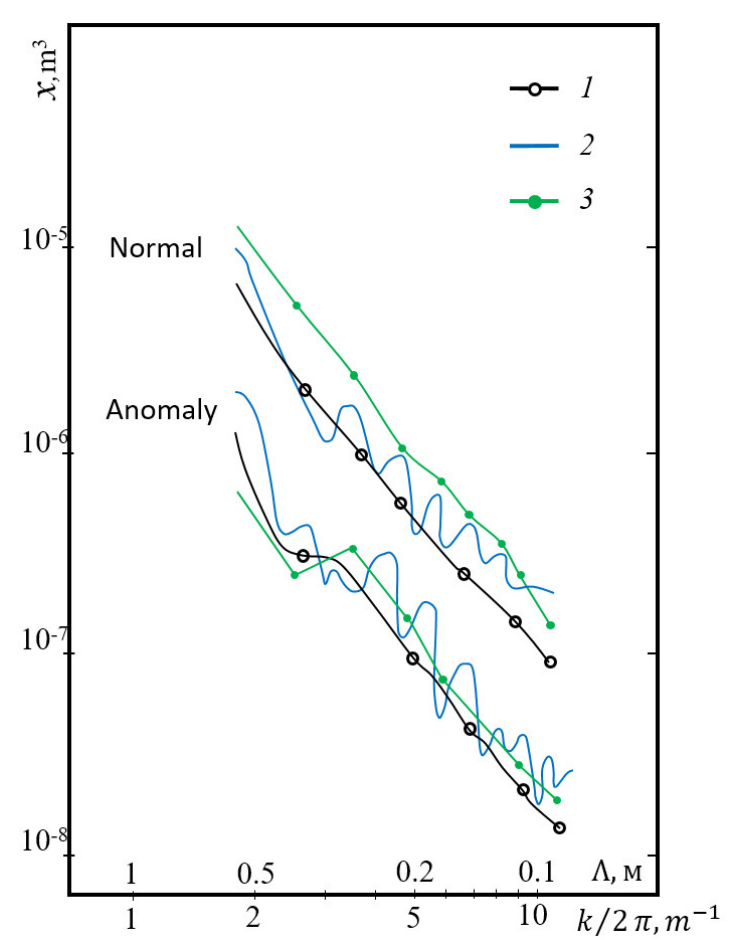

(b)

Figure 5. Spectra of sea surface elevations obtained by different methods [19]: (a) Average spectra during the experiment; (b) comparison of background spectra and spectra obtained in the presence of an anthropogenic anomaly. The figures indicate: 1 -retrieving from images by a nonlinear multiposition method; 2 -in situ measurements with a string wave recorder; 3-stereophotogrammetric measurements; red lines denote Toba approximation [26]. 
All the data presented can be divided into two classes-background and anomaly caused by oil on the water. If the background data are averaged over the results of all measurements of the data for all three methods, the rms elevation values given in Tables 1 and 2 will be obtained.

Table 1. Comparison of rms sea surface elevations measured by different techniques.

\begin{tabular}{ccccc}
\hline Class & $\begin{array}{c}\text { Range } \\
\lambda, \mathbf{m}\end{array}$ & $\begin{array}{c}\sigma_{\mathcal{\xi}}^{(\mathbf{R})}, \mathbf{m m} \\
\text { Non-Linear Retrieval }\end{array}$ & $\begin{array}{c}\sigma_{\mathcal{\xi}}^{(\mathrm{CT})}, \mathbf{m m} \\
\text { Stereo-Photography }\end{array}$ & $\begin{array}{c}\sigma_{\mathcal{\xi}}^{(\mathrm{K})}, \mathbf{m m} \\
\text { Wave-Recorder Measurements }\end{array}$ \\
\hline \multirow{2}{*}{ Background } & $0.1-1.0$ & 9.24 & 12.02 & 8.72 \\
& $0.04-0.4$ & 2.9 & 7.1 & 4.1 \\
\multirow{2}{*}{ Anomaly (Oil on water) } & $0.1-1.0$ & 3.30 & 6.00 & 3.98 \\
& $0.04-0.4$ & 2.0 & 2.4 & 2.9 \\
\hline
\end{tabular}

Table 2. The ratio of characteristics in the background and anomalous areas.

\begin{tabular}{ccccc}
\hline Ratio & $\begin{array}{c}\text { Range } \\
\lambda, \mathbf{m}\end{array}$ & Non-Linear Retrieval & Stereo-Photography & Wave-Recorder Measurement \\
\hline \multirow{2}{*}{ Ratio of rms elevations } & $0.1-1.0$ & 1.4 & 1.91 & 1.59 \\
& $0.04-0.4$ & 1.65 & 2.5 & 1.37 \\
Ratio of variances & $0.1-1.0$ & 1.96 & 3.64 & 2.51 \\
& $0.04-0.4$ & 2.72 & 6.25 & 1.88 \\
\hline
\end{tabular}

The tables also show the relationship between the background and the anomaly in the magnitude of the spectral power (squares of the root mean square elevation).

Thus, for the wavelength range from 0.1 to $1 \mathrm{~m}$, the rms elevation in the area of the sea surface anomaly associated with the appearance of an oil slick decreases:

- $\quad 1.4$-fold, according to the results of the developed technique;

- 1.91 -fold, based on the results of processing stereopairs;

- 1.59 -fold, according to the data of the in situ wave recorder.

Similarly, for the wavelength range from $0.04 \mathrm{~m}$ to $0.4 \mathrm{~m}$, the rms elevation in the area of the oil slick decreases:

- $\quad 1.65$-fold, according to the results of the developed technique;

- $\quad 2.5$-fold, according to the results of processing stereopairs;

- $\quad 1.37$-fold, according to the wave recorder data.

The obtained estimates of the spectral anomalies of the sea surface agree with the results of other researchers [22,27].

\section{Discussion}

The developed approach for retrieving the spatial spectra of slopes and elevations of surface waves from aerospace optical images takes into account the nonlinear modulation of the brightness field by the slopes of the sea surface and includes a number of computational operations, including the numerical synthesis of the slope fields of the sea surface with a given spatial spectrum and random phases, as well as modeling the brightness field, taking into account the conditions of illumination, reflection and light scattering.

In this case, parameterization of spatial-frequency filters using a set of parameters depending on the conditions of image registration can be used.

The conducted studies have demonstrated the adequacy of the application of the method for retrieving the spectra of sea wave slopes and elevations from space optical images of high spatial resolution, which takes into account the nonlinear nature of the modulation of the brightness field by slopes of the sea surface.

The possibility of using the developed method for studying the variability of sea waves in the coastal zone under conditions of limited fetch is shown. 
According to the results of two different complex experiments, the spatial spectral characteristics of sea waves, estimated from remote sensing data, were compared with the corresponding characteristics measured by in situ means under controlled conditions. The data of the array of string wave recorders installed on the stationary oceanographic platform of the Black Sea hydrophysical sea truth test site, as well as the data of stereo photography from the same platform, were used as sea truth data for comparison. Comparison of the wave spectra and its statistical characteristics demonstrated the correspondence of the results obtained during the processing of satellite images of high spatial resolution and the results of processing data obtained by various ground-based means. The measure of the discrepancy between the background wave spectra obtained from remote and in situ data is about 0.07. At the same time, there is a good qualitative agreement between the spectra obtained by distance and in situ methods in the area of an artificially created anomaly associated with the damping of short waves by an oil film on water.

Thus, the adequacy of remote measurement of both the time-average spectra of the sea surface and the variations of these spectra associated with unsteady waves has been experimentally confirmed. The use of high spatial resolution images and retrieving operators, taking into account the nonlinear nature of the modulation of the optical signal by the surface slopes, makes it possible to remotely determine the characteristics of wave spectra in a wide range of wavelengths and over vast areas.

The developed methods will be used for remote monitoring of the state of sea waves, including for identifying natural and anthropogenic impacts on various water areas.

At the same time, the studies carried out have shown the prospects of further development of the elaborated approach to retrieve wave spectra from optical satellite images using retrieving operators that take into account the nonlinear modulation of the brightness field by surface slopes.

A practically important direction in the development of the approach is its combination with other methods to reconstruct the angular distribution of spectral energy for all directions. In particular, a possible integration of the proposed method with the method for estimating the wave spectrum from the statistical characteristics of solar flares [23], as well as with developing new methods based on fractal analysis of optical satellite images [30] looks promising. The development and validation of new approaches will require new complex experiments using synchronous satellite and sea truth measurements.

\section{Conclusions}

The research results presented in this article and in the cited works indicate that the developed method to retrieve spatial spectra of slopes and elevations of surface waves from aerospace optical images, taking into account the nonlinear modulation of the brightness field by slopes of the sea surface, can be used for aerospace monitoring sea areas.

The developed retrieving operator can be used under the changing conditions of aerospace surveys and wave generation due to the use of spatial-frequency filter parameterization using a set of parameters that depend on the conditions of image registration.

As shown in this work, the proposed approach can be used to identify and study the variability of the sea wave spectra associated with anomalous phenomena and processes, including those of anthropogenic origin.

Author Contributions: Conceptualization, A.M. and V.B.; methodology, A.M.; software, A.M.; validation, A.M.; formal analysis, V.B.; investigation, A.M.; resources, V.B.; data curation, V.B.; writing—original draft preparation, A.M.; writing—review and editing, V.B.; visualization, A.M.; supervision, V.B.; project administration, V.B.; funding acquisition, V.B. Both authors have read and agreed to the published version of the manuscript.

Funding: The work was carried out with the financial support of the project by the Russian Federation represented by the Ministry of Science and Higher Education of the Russian Federation, the Agreement No. 075-15-2020-776.

Institutional Review Board Statement: Not applicable. The study did not involve humans or animals. 
Informed Consent Statement: Not applicable. The study did not involve humans.

Data Availability Statement: The study did not report or generate any data.

Acknowledgments: The authors are grateful to Vladimir A. Dulov for useful discussion and for cooperation in organization of experiments.

Conflicts of Interest: The authors declare no conflict of interest. The funders had no role in the design of the study; in the collection, analyses, or interpretation of data; in the writing of the manuscript, or in the decision to publish the results.

\section{References}

1. Bondur, V.G.; Grebenyuk, Y.V.; Sabinin, K.D. The spectral characteristics and kinematics of short-period internal waves on the Hawaiian shelf. Izv. Atmos. Ocean. Phys. 2009, 45, 598-607. [CrossRef]

2. Bondur, V.G.; Vorobjev, V.E.; Grebenjuk, Y.V.; Sabinin, K.D.; Serebryany, A.N. Study of fields of currents and pollution of the coastal waters on the Gelendzhik Shelf of the Black Sea with space data. Izv. Atmos. Ocean. Phys. 2013, 49, 886-896. [CrossRef]

3. Keeler, R.; Bondur, V.; Vithanage, D. Sea truth measurements for remote sensing of littoral water. Sea Technol. 2004, 45, 53-58.

4. Bondur, V.G. Satellite monitoring and mathematical modelling of deep runoff turbulent jets in coastal water areas. In Waste Water-Evaluation and Management; InTech: Rijeka, Croatia, 2011; pp. 155-180, ISBN 978-953-307-233-3. Available online: http:/ / www.intechopen.com/articles/show/title/satellite-monitoring-and-mathematical-modelling-of-deep-runoffturbulent-jets-in-coastal-water-areas (accessed on 28 July 2021). [CrossRef]

5. Bondur, V.G.; Zhurbas, V.M.; Grebenyuk, Y.V. Mathematical Modeling of Turbulent Jets of Deep-Water Sewage Discharge into Coastal Basins. Oceanology 2006, 46, 757-771. [CrossRef]

6. Phillips, O.M. Dynamics of the Upper Ocean Layer; Cambridge University Press: Cambridge, UK, 1977; 336p.

7. Collard, F.; Ardhuim, F.; Chapron, B. Routine monitoring and analysis of ocean swell fields using a spaceborne SAR. Geophys. Res. Lett. 2009, 36. [CrossRef]

8. Yurovskaya, M.V.; Dulov, V.A.; Chapron, B.; Kudryavtsev, V.N. Directional short wind wave spectra derived from the sea surface photography. J. Geophys. Res. 2013, 118, 4380-4394. [CrossRef]

9. Bondur, V.G.; Murynin, A.B. Methods for retrieval of sea wave spectra from aerospace image spectra. Izv. Atmos. Ocean. Phys. 2016, 52, 877-887. [CrossRef]

10. Bondur, V.G.; Dulov, V.A.; Murynin, A.B.; Yurovsky, Y.Y. A Study of Sea-Wave Spectra in a Wide Wavelength Range from Satellite and In-Situ Data. Izv. Atmos. Ocean. Phys. 2016, 52, 888-903. [CrossRef]

11. Bondur, V.G.; Dulov, V.A.; Murynin, A.B.; Ignatiev, V.Y. Retrieving Sea-Wave Spectra Using Satellite-Imagery Spectra in a Wide Range of Frequencies. Izv. Atmos. Ocean. Phys. 2016, 52, 637-648. [CrossRef]

12. Bondur, V.G.; Murynin, A.B. Measurement of Sea Wave Spatial Spectra from High-Resolution Optical Aerospace Imagery. In Surface Waves; Ebrahimi, F., Ed.; Intech: Rijeka, Croatia, 2018; pp. 71-88. Available online: https://www.intechopen. $\mathrm{com} /$ books/surface-waves-new-trends-and-developments/measurement-of-sea-wave-spatial-spectra-from-high-resolutionoptical-aerospace-imagery (accessed on 28 July 2021). [CrossRef]

13. Bondur, V.G.; Murynin, A.B. Vosstanovlenie spektrov poverkhnostnogo volneniya po spektram izobrazheniy s uchetom nelineynoy modulyatsii polya yarkosti (Restoration of surface waves spectra from the spectra of images with the account for nonlinear modulation of the brightness field). Opt. Atmos. Okeana 1991, 4, 387-393. (In Russian)

14. Murynin, A.B. Restoration of the spatial spectrum of the sea surface from the optical images in a nonlinear model of brightness field. Issled. Zemli Kosm. 1990, 6, 60-70. (In Russian)

15. Stilwell, D. Directional energy spectra of the sea from photographs. J. Geophys. Res. 1969, 74, 1974-1986. [CrossRef]

16. Monaldo, F.M.; Kasevich, R.S. Daylight Imagery of Ocean Surface Waves for Wave Spectra. J. Phys. Oceanogr. 1981, 11, $272-283$. [CrossRef]

17. Chapman, R.D.; Irani, G.B. Errors in estimating slope spectra from wave images. Appl. Opt. 1981, 20, 3645-3652. [CrossRef]

18. Murynin, A.B. Parameterization of filters retrieving the spatial spectra of sea surface slopes on the basis of optical imagery. Issled. Zemli Kosm. 1991, 5, 31-38. (In Russian)

19. Baranovskii, V.D.; Bondur, V.G.; Kulakov, V.V. Calibration of remote measurements of 2-D spatial wave spectra from optical images. Issled. Zemli Kosm. 1992, 2, 59-67. (In Russian)

20. Bondur, V.G.; Vorobyov, V.E.; Murynin, A.B. Retrieving Sea Wave Spectra Using High Resolution Satellite Imagery under Various Conditions of Wave Generation. Izv. Atmos. Ocean. Phys. 2020, 56, 887-897. [CrossRef]

21. Kosnik, M.V.; Dulov, V.A. Extraction of short wind wave spectra from stereo images of the sea surface. Measur. Sci. Technol. 2011, 22, 015504. [CrossRef]

22. Dulov, V.A.; Yurovskaya, M.V. Spectral Contrasts of Short Wind Waves in Artificial Slicks from the Sea Surface Photographs. Phys. Oceanogr. 2021, 28, 348-360. [CrossRef]

23. Kudryavtsev, V.; Yurovskaya, M.; Chapron, B.; Collard, F.; Donlon, C. Sun glitter imagery of ocean surface waves. Part 1: Directional spectrum retrieval and validation. J. Geophys. Res. Ocean. 2017, 122, 1369-1383. [CrossRef]

24. Yurovsky, Y.Y.; Dulov, V.A. MEMS-based wave buoy: Towards short wind-wave sensing. Ocean Eng. 2020, 217, 108043. [CrossRef] 
25. Preetham, A.J.; Shirley, P.; Smits, B.E. A practical analytical model for daylight. In Proceedings of the 26th Annual Conference on Computer Graphics and Interactive Techniques, SIGGRAPH 1999, Los Angeles, CA, USA, 8-13 August 1999; pp. 91-100. [CrossRef]

26. Toba, J. Local balance in the air-sea boundary process. Oceanogr. Soc. Jpn. 1973, 29, 209-225. [CrossRef]

27. Ermakov, S.A.; Salashin, S.G.; Panchenko, A.R. Film slicks on the sea surface and some mechanisms of their formation. Dyn. Atmos. Ocean. 1992, 16, 279-304. [CrossRef]

28. Ermakov, S.A.; Sergievskaya, I.A.; Da Silva, J.C.B.; Kapustin, I.A.; Shomina, O.V.; Kupaev, A.V.; Molkov, A.A. Remote Sensing of Organic Films on the Water Surface Using Dual Co-Polarized Ship-Based X-/C-/S-Band Radar and TerraSAR-X. Remote Sens. 2018, 10, 1097. [CrossRef]

29. Ermakov, S.A. Influence of Films on the Dynamics of Gravitational-Capillary Waves (Vliyanie Plenok na Dinamiku GravitacionnoKapillyarnyh Voln); Institute of Applied Physics, Russian Academy of Sciences: Nizhnij Novgorod, Russia, 2010; 165p.

30. Murynin, A.B.; Richter, A.A.; Shakhramanyan, M.A. Selection of Informative Features to Highlight Areas of Waste Disposal by High Spatial Resolution Satellite Imagery. Izv. Atmos. Ocean. Phys. 2019, 55, 1403-1415. [CrossRef] 